\title{
Inelastic deformation behavior of thermal barrier coatings exposed at a high-temperature environment
}

\author{
M. Arai ${ }^{1, \mathrm{a}}$, and W. $\mathrm{Wu}^{2}$ \\ ${ }^{1}$ Materials Science Research Lab., Central Research Institute of Electric Power Industry, 2-6-1 \\ Nagasaka, Yokosuka-shi, Kanagawa-ken, Japan \\ ${ }^{2}$ School of Aerospace, Xi' an Jiaotong University, Xi' an 710049, China
}

\begin{abstract}
Thermal barrier coatings (TBCs) are usually deposited onto the surface of the high-temperature component such as gas turbine, in order to protect it from a hightemperature environment. Coating stress generated by such a high-temperature brings serious damages in TBCs in service. For predicting numerically it, it is necessary to develop the constitutive equation suite to plasam-sprayed TBCs. Previous studies have made clear that the freestanding ceramic coat peeled from TBC coated substrate deforms nonlinearly with a mechanical loading, however the results there have been restricted to the test done using as-sprayed sample. In this study, effect of deposition parameter and high-temperature exposure condition on stress-strain curve of the freestanding ceramic coating sample was examined. The associated deformation process was discussed with the microstructure changes observed after performing a bending test for the exposed sample.
\end{abstract}

\section{Introduction}

Ceramic thermal barrier coatings (TBCs) are an important technology for protecting hightemperature components of a land-based gas turbine from an aggressive environment. TBCs are generally deposited using a plasma-spray technique. This deposition technology is known as a mechanical process impacting and depositing continuously particles molten into a plasma hightemperature flow onto a target surface [1]. Thus, a stress-strain curve as one of the mechanical properties of the ceramic coating in TBCs is affected strongly by the deposition parameter.

Previous studies [2] have been made clear that stress-strain curve obtained performing a bending test indicates a nonlinear behavior in spite of ceramic material, and a lower particle velocity as one in the deposition condition brought more strong nonlinear behavior. SEM observation has revealed that a nonlinear deformation progressed with in-plane slip in the microcrack formed at boundary between flatten particles; it is called as splat boundary.

Now, TBC coated components in service are subjected to a high-temperature environment, and it could bring about serious damages such as cracking and delamination of TBC. Thus, it is also important to know about effect of a high-temperature exposure condition on mechanical properties of the ceramic coating in TBCs. The purpose of this study is to examine about influence of an

\footnotetext{
a e-mail : marai@ criepi.denken.or.jp
} 
isothermal exposure condition on stress-strain curve as one of the mechanical properties of the ceramic coating in TBCs. For achieving it, we perform a bending test using the isothermally exposed freestanding ceramic coating sample according to the previous experimental procedure [2]. After the bending test, the microstructure is examined by a scanning electron microscope (SEM) in order to identify the deformation mechanism progressed in the coating sample exposed for a long period.

\section{Coating preparation}

$8 \mathrm{wt} \% \mathrm{Y}_{2} \mathrm{O}_{3}-\mathrm{ZrO}_{2}$ (Sulzer Metco, 204NS) for ceramic coating and CoNiCrAlY (Praxair, CO-210-1) for metallic bond coating were employed as spraying powders. Type 304 stainless steel plate with geometry $100 \times 50 \times 5 \mathrm{~mm}$ was prepared as the underlying substrate.

TBC was deposited onto the substrate by following process. Firstly, CoNiCrAlY was deposited with thickness $0.1 \mathrm{~mm}$ by using atomospheric plasma spraying (APS) technique. Ceramic coating was then deposited using the same device. In this study, particle energy was controlled as the deposition parameter; low, middle and high energies. The associated particle velocity was identified with DPV 2000 (Tecnar Automation). The coating thickness was also changed with 0.5 and $1.0 \mathrm{~mm}$ as the other deposition parameter.

The TBC-coated plate was cut in the geometry of $4.5 \times 25 \mathrm{~mm}$ using a diamond cutter. In order to obtain a freestanding ceramic coating sample, both CoNiCrAlY and the substrate were dissolved using an electrolytic polishing process. This sample was employed for performing a bending test.

\section{Experimental procedure}

Stress-strain curve for the freestanding ceramic coating sample was obtained by performing a cantilever bending test. The bending test was done using a handmade bending tester (see Fig.1). The distance between support and load points was taken as $18 \mathrm{~mm}$. A bending load was measured by a stiff load cell (LUR-A-100N; Kyowa dengyo), and a strain was also measured by a strain gage (KFG-2-120-C1-11L1M2R; Kyowa dengyo) attached at tension side at center of the sample surface. Both load and strain were monitored and stored continuously in a personal computer via A/D converter (NR-2000; KYENCE). The bending load versus the strain was transferred to stress-strain curve using a bending formula in basis of classic beam theory.

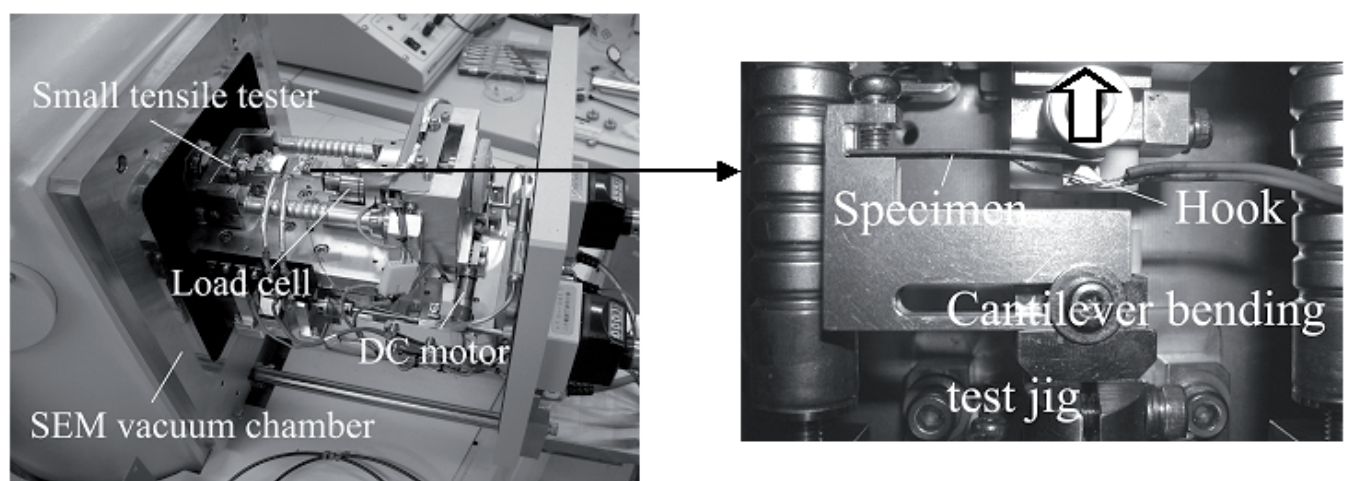

Fig.1 Appearance of small bending tester combined with SEM, which was developed by our group. Right picture indicates a detail around the bending test part. 


\section{Experimental results and discussion}

\subsection{Stress-strain curve}

Figure 2 indicates a typical stress-strain curve of the freestanding ceramic coating sample obtained by performing the bending test. Those results were obtained using the coating sample deposited under the deposition condition of particle velocity $150.3 \mathrm{~m} / \mathrm{s}$, viz. low energy level in particle energy, the coating thickness $0.5 \mathrm{~mm}$ and also the exposure time 500 hours. The stress-strain curve shows obviously a nonlinear deformation behavior in both the as-sprayed and the isothermally exposed samples. The comparison of a stress level reveals that the stiffness in the isothermally exposed sample becomes softer than the as-sprayed one. However, if we have attention in the exposed sample only, it can be recognized that a lower exposure temperature condition leads to more soft response.

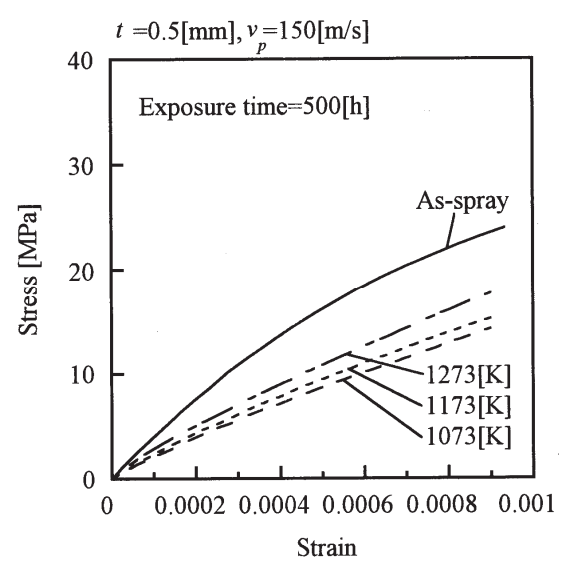

Fig.2 Typical stress-strain curve obtained by performing the bending test for the ceramic coating.

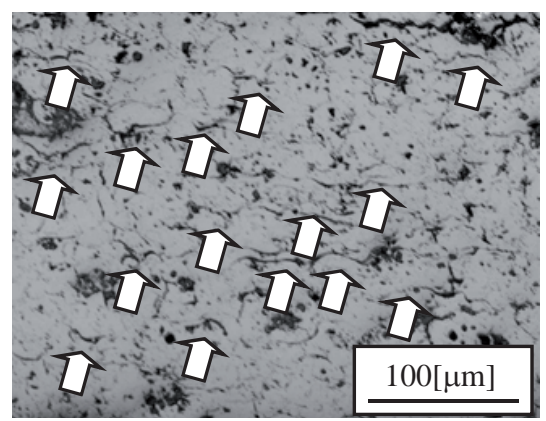

(a)Cross section of the sample extracted from TBC sample exposed at $1273 \mathrm{~K}$ for $500 \mathrm{~h}$.

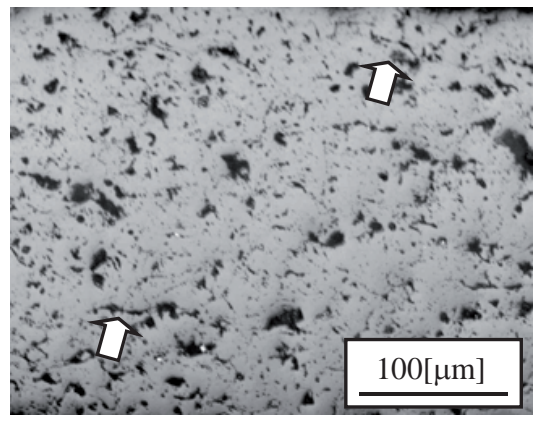

(b)Cross section of the sample exposed for the freestanding ceramic coating at $1273 \mathrm{~K}$ for $500 \mathrm{~h}$.

Fig. 3 SEM observation resutls for cross section of the ceramic coating after isothermal exposure.

Arrow indicates microcrack formed along splat boundary.

\subsection{Microstructure characterization}

Figure 3 shows microstructure observed for a cross section of the ceramic coating. Figure 3(a) is the sample extracted electrochemically from TBC-coated sample exposed at $1273 \mathrm{~K}$ for 500 hours, and 
also Fig. (b) is the picture taken after exposing the freestanding ceramic coating at the same temperature condition. Both microstructures include a lot of globular pores and microcracks at splat boundary as known as a typical microstructure in plasma-sprayed TBCs. However, if we notice about number of microcracks, it is understood that number of microcracks counted in Fig. (a) is much larger than one in Fig. (b). The difference appeared in both samples would be caused by a thermal stress formed by thermal expansion mismatch between the ceramic coating and the underlying substrate during the isothermal exposure. Namely, a large tensile thermal stress was occurred in case of Fig. (a), which led to many microcracks in the microstructure. In this study, we attend to microcrack density instead of number of microcracks, in order to characterize microdamage induced mechanically and/or thermally in the ceramic coating microstructure. In next section, we will relate microcrack density and deformation response such as elastic modulus and nonlinear degree in the stress-strain curve.

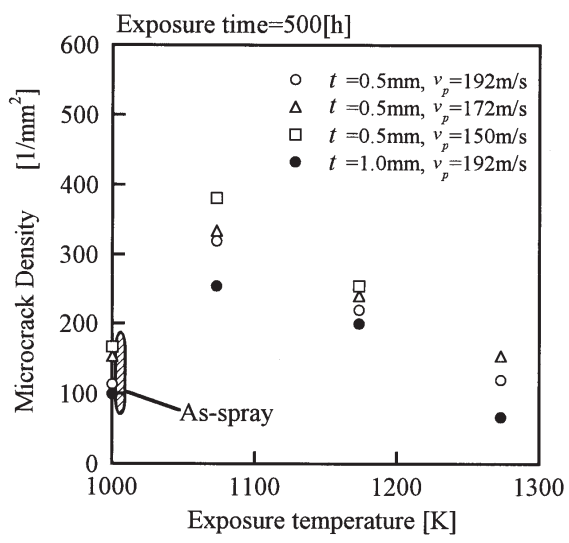

(a) Influence of exposure temperature

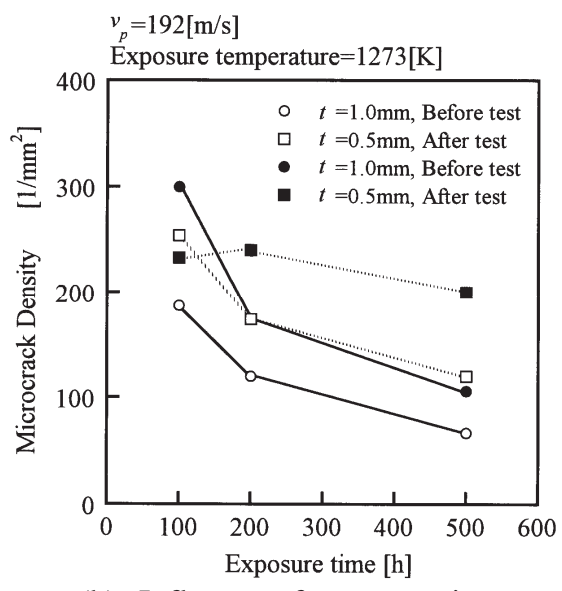

(b) Influence of exposure time

Figure 4 Influence of the exposure condition on microcrack density.

\subsection{Relationship between microcrack density and deformation response}

Figure 4 shows relationship between microcrack density and the exposure condition. The microcrack density here was defined by dividing number of cracks by SEM picture area. Figure 4(a) is the result in exposure temperature. Fistly, having attention in the case for the coating thickness $0.5 \mathrm{~mm}$ and 
particle velocity $192 \mathrm{~m} / \mathrm{s}$, the microcrack density for the sample exposed at $1073 \mathrm{~K}$ is larger than one for the as-sprayed sample, and is then decreased with the elevating exposure temperature. Otherwise, the microcrack density increases with decreasing particle velocity. The reason why the former trend was occurred is originated at both thermal stress and sintering phenomena; microcrack in the assprayed microstructure was increased by experiencing a high-temperature exposure environment, viz. generating a tensile thermal stress in the ceramic coating, and then decreases with a sintering process as the exposure time is longer.

Figure 4(b) is the case in exposure time. This graph also includes the results in microcrack density counted before and after bending tests. It is found that the microcrack density decreases with increase of the exposure time. Otherwise, comparing with microcrack densities counted before and after bending tests, the microcrack density observed in the sample after the bending test is larger than one before the test. Thus, this comparison reveals that a tensile loading enforced to the ceramic coating by any mechanical tests leads to increase of number of microcracks, in addition to thermal stress effect.

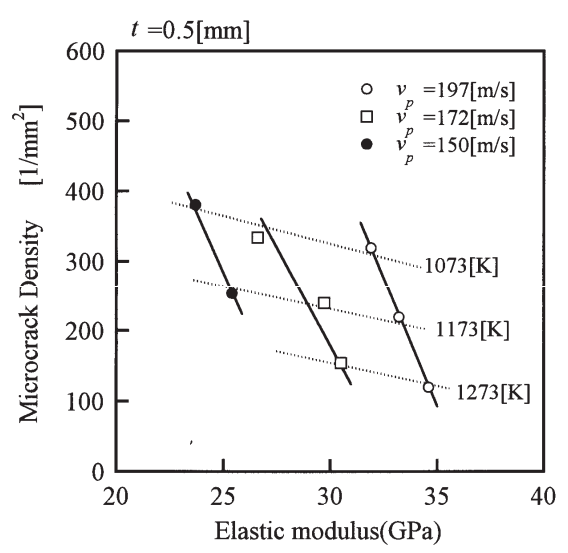

Figure 5 Relationship between microcrack density and elastic modulus.

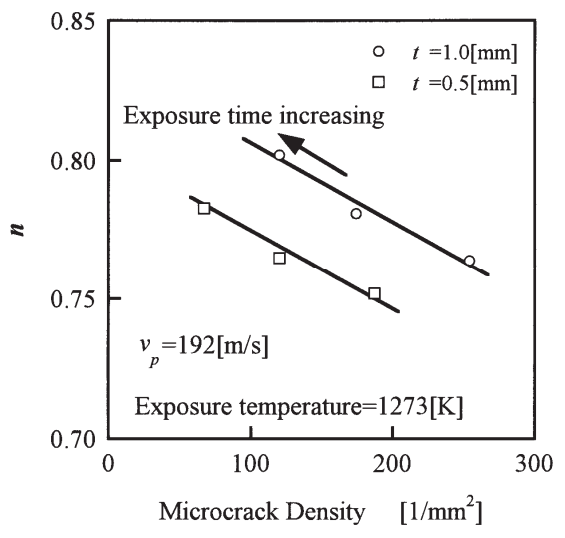

Figure 6 Relationship between exponent $n$ and microcrack density.

Now, let's relate between microcrack density and mechanical properties of the ceramic coating in order to discuss inelastic deformation mechanism appeared in a plasma-sprayed ceramic coating. Figure 5 indicates relationship between microcrack density and elastic modus that was defined as a tangent at origin in the stress-strain curve. Those results include the sample with the coating thickness $0.5 \mathrm{~mm}$ and the exposure time $500 \mathrm{~h}$. Gray line in the graph shows the line which was obtained by connecting between symbols at the same exposure temperature, and solid line is one 


\section{EPJ Web of Conferences}

connected symbols with the same particle velocity. The microcrack density changes linearly with the deposotion parameter and the exposure condition; that is, the microcrack density decreases with increasing particle velocity and elevating exposure temperature. Otherwise, such decrease of microcrack density leads to increase of elastic modulus. This trend is reasonable in view of deformation analysis in elastic solid included microcracks in micromechanics.

Figure 6 indicates relationship between exponent $n$ and the microcrack density. The exponent characterizes a nonlinear degree in a stress-strain curve, and is defined as $n$ included in the simple plastic constitutive equation: $\sigma=A \varepsilon^{n}$. If this value goes to 1 , associated stress-strain response approaches to elastic response. Those results were obtained using the sample with particle velocity $192 \mathrm{~m} / \mathrm{s}$ and exposure temperature $1273 \mathrm{~K}$. The exponent value increases with the exposure time. That is, the deformation response of the ceramic coating sample becomes stiff via a longer exposure time, which was caused by a sintering process. Otherwise, associated microcrack density decreases with the increasing exponent value.

The microstructure characterization revealed about deformation mechanism of the plasmasprayed ceramic coating that the elastic modulus is decreased by microcracks induced by thermal stress when a high-temperature environment being exposed, and then inelastic deformation is progressed with a mechanical loading because of more increased number of microcracks in addition of in-plane sliding along splat boundary. Particle velocity in the deposition parameter affects strongly mechanical properties of the ceramic coat, viz. a lower particle velocity leads to lower elastic modulus. Otherwise, a long exposure time makes the coating a stiff material for encouraging closure of microcracks by a sintering

\section{Conclusion}

In this study, influence of isothermal exposure condition on inelastic deformation in plasma-sprayed ceramic coating was examined via a bending test. As obtained results, the freestanding ceramic coating sample exposed at a high-temperature indicated a nonlinear behavior as well as observing in the as-sprayed one. It was found that the exposure condition affects elastic modulus and nonlinear degree $n$; elastic modulus increases with higher particle velocity as the deposition parameter and also elevating exposure temperature in the exposure condition. Nonlinear degree characterizing a nonlinear stress-strain curve increases with longer exposure time, viz. approaches to 1 as known as elastic case. This trend could be related with microcrack density including a microstructure.

\section{References}

1. M. Arai, E. Wada, K. Kishimoto, J. of Solid Mech. and Mat. Eng. 1, 10 (2007), pp.1251-1261.

2. M. Arai, W. Wu, K. Fujimoto, J. of Solid Mech. and Mat. Eng. in press. 